\title{
The Gut Microbial Community of Antarctic Fish Detected by $16 S$ rRNA Gene Sequence Analysis
}

\author{
Wei Song, Lingzhi Li, Hongliang Huang, Keji Jiang, Fengying Zhang, Xuezhong Chen, \\ Ming Zhao, and Lingbo Ma
}

Key Laboratory of East China Sea and Oceanic Fishery Resources Exploitation, East China Sea Fisheries Research Institute, Chinese Academy of Fishery Sciences, Ministry of Agriculture, Shanghai, China

Correspondence should be addressed to Lingbo Ma; swift83@sina.com

Received 29 July 2016; Accepted 9 October 2016

Academic Editor: Pengjun Shi

Copyright (C) 2016 Wei Song et al. This is an open access article distributed under the Creative Commons Attribution License, which permits unrestricted use, distribution, and reproduction in any medium, provided the original work is properly cited.

Intestinal bacterial communities are highly relevant to the digestion, nutrition, growth, reproduction, and a range of fitness in fish, but little is known about the gut microbial community in Antarctic fish. In this study, the composition of intestinal microbial community in four species of Antarctic fish was detected based on 16S rRNA gene sequencing. As a result, 1004639 sequences were obtained from 13 samples identified into 36 phyla and 804 genera, in which Proteobacteria, Actinobacteria, Firmicutes, Thermi, and Bacteroidetes were the dominant phyla, and Rhodococcus, Thermus, Acinetobacter, Propionibacterium, Streptococcus, and Mycoplasma were the dominant genera. The number of common OTUs (operational taxonomic units) varied from 346 to 768, while unique OTUs varied from 84 to 694 in the four species of Antarctic fish. Moreover, intestinal bacterial communities in individuals of each species were not really similar, and those in the four species were not absolutely different, suggesting that bacterial communities might influence the physiological characteristics of Antarctic fish, and the common bacterial communities might contribute to the fish survival ability in extreme Antarctic environment, while the different ones were related to the living habits. All of these results could offer certain information for the future study of Antarctic fish physiological characteristics.

\section{Introduction}

Although the Southern Ocean occupies $10 \%$ of the world's ocean, only 322 species fishes in Antarctic Ocean were recognized currently, considered so small comparing to the global diversity approximately $25,000 \sim 28,000$ species, while the benthic fish fauna includes 19 families of about 222 species [1].

Antarctic fishes have been isolated for over 10 million years. Besides, they have developed mechanisms to adapt to and survive in the coldest and most thermally stable environment now [2-5]. Antarctic water under the sea ice possesses a very low and fairly constant temperature of about $-1.86^{\circ} \mathrm{C}$, and annual temperature fluctuations are in $1^{\circ} \mathrm{C}$; Antarctic sea water is with a high oxygen concentration of $0.18-0.36 \mathrm{mmol} / \mathrm{L}$, which enables ice fish to live with no haemoglobin [6-8].

Trematomus bernacchii (family Notothenioidei), Chionodraco hamatus, (family Channichthyidae), Gymnodraco acuticeps (family Bathydraconidae), and Pagothenia borchgrevinki (family Notothenioidei) are four Antarctic fish living in the oxygen-rich coastal Antarctic Ocean with the equilibrium temperature at $-1.86^{\circ} \mathrm{C}$ year round $[2,9]$. T. bernacchii, C. hamatus, and G. acuticeps are benthic fish and always live in the depth of more than $100 \mathrm{~m}$ under the surface of the sea ice, while $P$. borchgrevinki likely live in the water $1 \sim 2 \mathrm{~m}$ below the ice [10-13].

Fish have stable microbiota in the gastrointestinal (GI) tract, and the microbiota considered as an integral part of the host is highly relevant to the digestion, nutrition, growth, and reproduction and strongly affects fish health by assisting the gut epithelium development and stimulating the innate immune system $[14,15]$. Furthermore, some papers have provided that the microbes living in the fish intestines are influenced by dietary manipulations [16] while little is known about the bacterial community composition of Antarctic fish and whether the Antarctic fish gut bacterial community was affected by extreme environmental conditions. 
16S rRNA-based molecular methodologies are now commonly used for identifying and classifying the bacterial species within compounded microbial communities [17]. In this study, we used Illumina MiSeq platform and comparative sequence analysis to determine the microbial diversity of $T$. bernacchii, C. hamatus, G. acuticeps, and P. borchgrevinki.

\section{Materials and Methods}

2.1. Sample Collection and Preparation. A total of thirteen fish belonging to four species, four of T. bernacchii, two of $C$. hamatus, five of G. acuticeps, and two of P. borchgrevinki, were used. All of these fish were caught at 100-200 m under the ice by net near the location of $72^{\circ} 55^{\prime} \mathrm{E}$ and $67^{\circ} 29^{\prime} \mathrm{S}$ through Chinese Antarctic research vessel Xue Long.

Experimental fish were randomly harvested with net and then euthanized by an overdose of MS 222. After dissection, the intestines were removed aseptically from each fish abdominal cavity; the contents were carefully collected and labelled Tb1-Tb4 for T. bernacchii, Ch1-Ch5 for C. hamatus, Ga1-Ga2 for G. acuticeps, and Pb1-Pb2 for P. borchgrevinki and stored at $-80^{\circ} \mathrm{C}$ before transporting to the laboratory.

2.2. DNA Extraction. The intestinal content samples were thawed on ice, and then genomic DNA were separately extracted using the E.Z.N.A. Stool DNA kit (OMEGA, BioTek, USA) based on the manufacturer's protocol and stored at $-80^{\circ} \mathrm{C}$. The integrity of the $13 \mathrm{DNA}$ samples was assessed visually using agarose gel (containing ethidium bromide) electrophoresis on $1.0 \%$ and quantified using a Qubit v2.0 fluorometer (Life Technologies, Darmstadt, Germany). The DNA concentration was determined by using a fluorescence spectrophotometer (ES-2, Malcom, Japan).

2.3. PCR Amplification and Sequencing. The hypervariable regions V4-V5 of the $16 \mathrm{~S}$ rRNA gene were amplified using a universal primer set $515 \mathrm{~F}$ (5-GTGCCAGCMGCCGCGG-3) and 907 R (5-CCGTCAATTCMTTTRAGTTT-3). The PCRs were performed in triplicate using $25 \mu \mathrm{L}$ reaction system with $1 \mu \mathrm{L}$ each primer $(10 \mu \mathrm{M}), 2 \mu \mathrm{L}$ DNA template $(20 \mathrm{ng} / \mu \mathrm{L})$, $5 \mu \mathrm{L} 5 \mathrm{x}$ Q 5 reaction buffer, $5 \mu \mathrm{L} 5 \mathrm{x}$ Q5 GC high enhancer, $2 \mu \mathrm{L}$ dNTPs $(2.5 \mathrm{mM})$, and $0.25 \mu \mathrm{L}$ Q 5 polymerase $(5 \mathrm{U} / \mu \mathrm{l})$. The PCR amplification conditions were 1 cycle of $98^{\circ} \mathrm{C}$ for $3 \mathrm{~min}$ (initial denaturation), followed by 25 cycles of $98^{\circ} \mathrm{C}$ for $15 \mathrm{~s}$ (denaturing), $50^{\circ} \mathrm{C}$ for $30 \mathrm{~s}$ (annealing) and $72^{\circ} \mathrm{C}$ for $30 \mathrm{~s}$ (extension), and finally 1 cycle of $72^{\circ} \mathrm{C}$ for $5 \mathrm{~min}$ (final extension). The amplified PCR products were examined by $2 \%$ gel electrophoresis, purified by using the MinElute Gel Extraction Kit (Qiagen) to remove the unspecific DNA fragments and quantitated by using Bioanalyzer 2100 (Agilent Technologies, Waldbronn, Germany). The products were pooled together with equal amount and sequenced on the Illumina MiSeq platform (Roche Applied Science, Indianapolis, IN, USA). And the length of paired-end reads was $150 \mathrm{bp}$.

2.4. Data and Statistical Analysis. The raw sequences obtained from Illumina MiSeq were firstly filtered for quality control and reads with length $<150 \mathrm{bp}$, ambiguous bases, average base quality score of $<20$ in the tags were discarded. Then FLASH (version 1.2.7, http://ccb.jhu.edu/software/FLASH/) was used to merge readl and read2 [18], and sequences with overlap $<10$ and mismatches were removed. After that, Quantitative Insights Into Microbial Ecology (QIIME) (version 1.9.0, http://qiime.org/) [19] was used to the further quality control and uchime of mothur (version 1.31.2) [20, 21] was used for chimera checking.

Reads after quality control were delineated into operational taxonomic units (OTUs) with a $97 \%$ sequence similarity using uclust of QIIME, and OTUs with abundance less than $0.001 \%$ of the total sequences were discarded. The taxonomic information of the representative sequence in each OTU was obtained by matching sequence database using BLAST of QIIME.

The rarefaction curves and bar graph of species distribution for the 13 samples were constructed using QIIME and the alpha-diversity indices (i.e., Chaol estimator and Shannon estimator) were calculated using mothur. The analysis of shared and unique operational taxonomy unit (OTU) between the four species was conducted based on the OTU table generated by the QIIME (v1.9.0).

To compare the similarity of the microbial community composition among the 13 intestinal contents of the four species of Antarctic fish, difference of microbial community in each sample was calculated by the Principal Components Analysis (PCA) and the heatmap associated with evolutionary relationship among different samples was also constructed and analyzed.

\section{Results}

3.1. The Microbial Complexity. A total of 1061710 sequences were obtained from 13 samples with the number of sequences ranging from 28296 to 138254 per individual after filtering for quality. By removing chimeras, 26978 to 119888 sequences were collected from each sample, resulting in a total of 1004639 sequences from all samples. Then all the sequences were clustered into 2199 OTUs at the $97 \%$ sequence similarity value (Table 1 ).

The microbial complexities in the gut of T. bernacchii, $C$. hamatus, G. acuticeps, and $P$. borchgrevinki were estimated on the basis of alpha-diversity indices (Chaol indices and Shannon indices). The Chaol was used to estimate species richness, while Shannon's index was used to indicate species diversity. The results showed that $C$. hamatus samples had the largest alpha-diversity indices followed by G. acuticeps, T. bernacchii, and P. borchgrevinki (Table 2).

3.2. Microbial Community Composition. After sampling 20000 reads, with the sampled read number increasing, the newly discovered OTUs reduced and the rarefaction curves tended to attain the saturation plateau (Figure 1). This showed that the libraries of the 13 samples were large enough to estimate the phylotype richness and microbial community diversity at the $97 \%$ similarity threshold.

All sequences were identified into 36 phyla, and only $0.6 \%$ of the total sequences were assigned to unspecified microbial phyla. Phyla with abundance $>0.1 \%$ of the 13 samples were 
TABLE 1: Sequence and taxonomy number of individual sample.

\begin{tabular}{|c|c|c|c|c|c|c|c|}
\hline Samples & Effective sequence & High quality sequence & Phylum & Class & Order & Family & Genus \\
\hline Tb1 & 39021 & 37534 & 433 & 432 & 427 & 373 & 236 \\
\hline $\mathrm{Tb} 2$ & 28296 & 27392 & 321 & 321 & 318 & 276 & 163 \\
\hline $\mathrm{Tb} 3$ & 52250 & 49982 & 662 & 659 & 652 & 581 & 352 \\
\hline $\mathrm{Tb} 4$ & 28698 & 26978 & 449 & 447 & 440 & 381 & 190 \\
\hline $\mathrm{Pbl}$ & 53101 & 49930 & 527 & 525 & 514 & 452 & 249 \\
\hline $\mathrm{Pb} 2$ & 61641 & 59218 & 642 & 640 & 634 & 571 & 375 \\
\hline Ch1 & 49882 & 47539 & 719 & 717 & 698 & 612 & 413 \\
\hline Ch2 & 39837 & 36146 & 776 & 776 & 768 & 616 & 377 \\
\hline Ch3 & 75228 & 71669 & 865 & 863 & 855 & 677 & 401 \\
\hline Ch4 & 64234 & 60660 & 798 & 796 & 788 & 633 & 379 \\
\hline Ch5 & 40188 & 37524 & 616 & 614 & 606 & 538 & 350 \\
\hline Gal & 59050 & 57647 & 616 & 616 & 607 & 521 & 338 \\
\hline $\mathrm{Ga} 2$ & 29089 & 27200 & 335 & 335 & 333 & 301 & 207 \\
\hline Total & 1061710 & 1004639 & & & & & \\
\hline
\end{tabular}

TABLE 2: The alpha indices of different samples.

\begin{tabular}{lcccc}
\hline Samples & Chao1 $^{\mathrm{a}}$ & ACE & Simpson & Shannon \\
\hline Tb1 & 680.5789 & 590.8154 & 0.782606 & 3.728343 \\
Tb2 & 593.1923 & 717.972 & 0.405413 & 2.07083 \\
Tb3 & 862.28 & 827.5564 & 0.928014 & 5.277833 \\
Tb4 & 664.5172 & 542.6397 & 0.955801 & 5.769862 \\
Pb1 & 845.8936 & 734.8494 & 0.905419 & 4.801013 \\
Pb2 & 890.375 & 847.3593 & 0.910085 & 5.060302 \\
Ch1 & 908.3 & 867.9615 & 0.918179 & 5.479842 \\
Ch2 & 891.25 & 850.9911 & 0.941227 & 6.02579 \\
Ch3 & 1004 & 948.9715 & 0.894694 & 5.562919 \\
Ch4 & 983.7581 & 927.8588 & 0.934068 & 6.029751 \\
Ch5 & 886.8971 & 848.5095 & 0.927018 & 5.530419 \\
Ga1 & 756.8333 & 706.2764 & 0.892472 & 4.79657 \\
Ga2 & 551.7576 & 504.3151 & 0.800829 & 3.598119 \\
\hline
\end{tabular}

clearly observed in the bar graph of species distribution (Figure 2). Proteobacteria (30.8\%), Actinobacteria (29.8\%), Firmicutes (13.7\%), Thermi (7.6\%), and Bacteroidetes (6\%) were the most dominant groups which accounted for $87.90 \%$ of the reads and commonly observed in all 13 fish guts. Other major phyla, including Tenericutes (3.6\%), Crenarchaeota $(2.8 \%)$, and Cyanobacteria (1.8\%), were also identified in all fish samples, but Crenarchaeota, Parvarchaeota (1.6\%), and Euryarchaeota were the only three phyla belonging to Archaea, and the latter two were only present in Chionodraco hamatus. Though the major bacterial phyla in the 13-fish intestinal content were similar, the relative abundance was obviously different.

At the genus level, the sequences from 13 samples were identified into 804 genera ranging from 102 to 210 per individual. The gut content samples were dominated by six major genera, representing approximately $49.3 \%$ of the sequences, including Rhodococcus (19.5\%), Thermus (7.5\%), Acinetobacter (7.1\%), Propionibacterium (6.5\%), Streptococcus
(5.1\%), and Mycoplasma (3.6\%). All above genus and Corynebacterium (1.8\%) and Flavobacterium (1.3\%) were present in all intestinal content samples.

3.3. Common and Unique Microbial Communities. The analysis of the common and unique OTUs was conducted to investigate the gut microbial community in different fish through a Venn diagram. Pairwise comparison was performed among the four species fish via considering the shared OTUs, as those present in a certain percentage at least $30 \%$ or $40 \%$ of the samples of each species fish gut, and the unique OTUs were defined as those only present in more than $30 \%$ or $40 \%$ of the samples taken from one species of fish gut sample and unfound in the other three species of fish gut samples.

The number of common OTUs ranged from 346 to 768 and unique OTUs varied from 84 to 694 (Figure 3). Many OTUs were uniquely present in only one species of fish gut sample, especially in T. bernacchii (about 694), while the common OTUs in the four species of fish gut were not a few yet (over 346). 


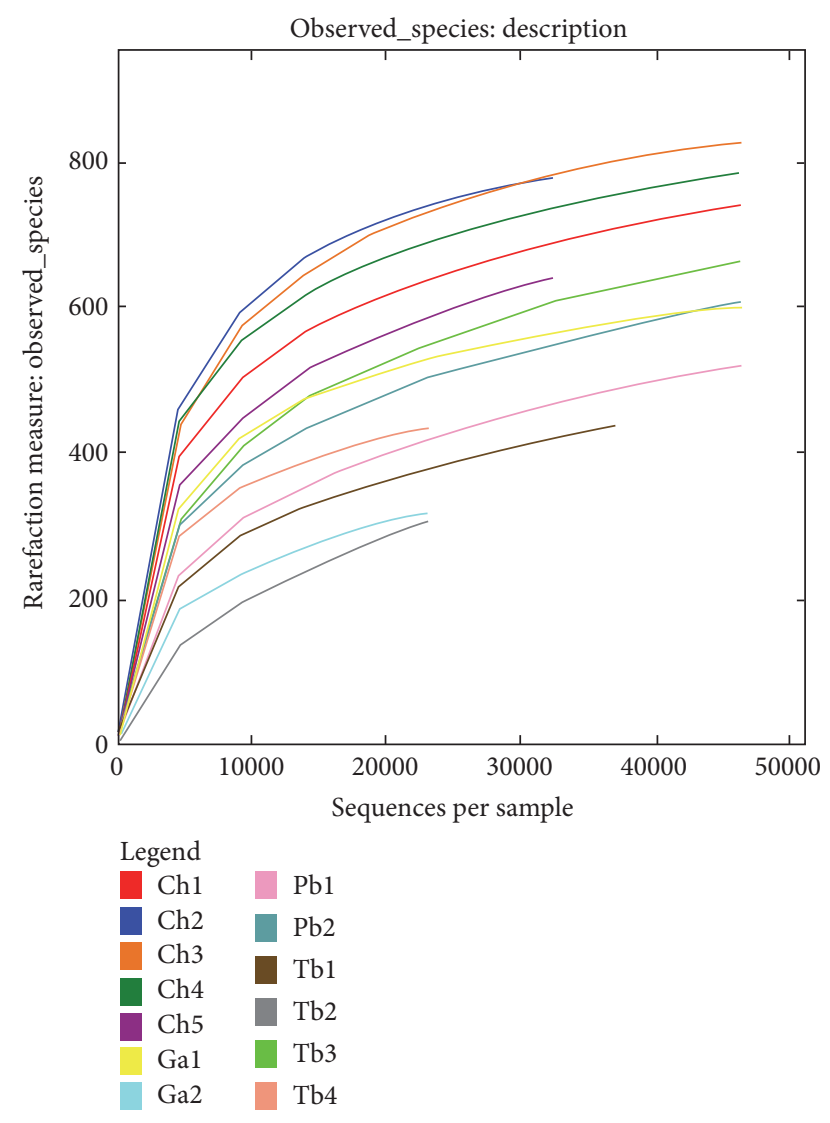

FIgURE 1: Rarefaction curve.

3.4. The Similarity of Microbial Community Composition. The similarity and difference of microbial community compositions of 13 intestinal content samples taken from the four species of Antarctic fish (T. bernacchii, C. hamatus, G. acuticeps, and P. borchgrevinki) were showed in PCA plot with $\mathrm{PC1}$ accounting for $38.78 \%$ of the total variation and PC2 for $17.83 \%$. As a result, no species, except 3 C. hamatus samples and $1 \mathrm{~T}$. bernacchii sample, formed a distinct cluster and clearly separated from other three species (Figure 4).

The hierarchically clustered heatmap analysis associated with the similarity of microbial community composition was performed at the genus level disclosing the richness and diversity of bacterial communities in the gut content of each sample. The composition of intestine microbiota could not show obvious similarity based on each species of Antarctic fish, but for individual, Ph2, Ch1, Ch5, and Gal showed higher similar, Ch2, Ch3, and Ch4 had higher similarity, Tb1, $\mathrm{Tb} 2, \mathrm{~Tb} 3$, and Ga2 had closer relationship, and $\mathrm{Tb} 4$ and $\mathrm{Pb} 1$ showed higher similarity (Figure 5).

\section{Discussion}

As is well known, the habitat is a key factor for the survival of organisms, and living temperature represents a significant driving force for biological evolution. Evolution of Southern Ocean organism occurred accompanied by the striking albeit intermittent temperature for about 60 million years [22]. The habitat modifications force the fish fauna to develop a number of morphological and physiological adaptations in order to survive in a cold, highly oxygenated environment without hematocrit and hemoglobin [23, 24].

T. bernacchii, C. hamatus, G. acuticeps, and P. borchgrevinki are four order Perciformes and important species in the Antarctic Ocean. Although studies on fish intestinal microbiota have been reported and the mechanisms of the survival ability about Antarctic fish have been shown in many papers, little is still known about the intestinal microbial community in Antarctic fish [25-29].

This study aims to detect the composition of intestinal microbial community in four species of Antarctic fish based on 16S rRNA gene sequence through Illumina MiSeq platform. As a result, 1004639 sequences were obtained and clustered into 2199 OTUs based on 97\% sequence similarity level and identified into 36 phyla and 804 genera for the 13 samples, showing a large microbial diversity in the Antarctic fish. Actinobacteria, Proteobacteria, Firmicutes, Thermi, and Bacteroidetes were the most dominant groups at phylum level, and Rhodococcus, Thermus, Acinetobacter, Propionibacterium, Streptococcus, and Mycoplasma were more abundant in the genus level. The result that Proteobacteria and Firmicutes act as dominant groups at phylum level is consistent with the previous finding [29]. Firmicutes and Bacteroidetes contribute to carbohydrates and/or proteins fermentation in the intestine to help the host acquire nutrients from the diet [30]. Crenarchaeota was presented in the four species and accounted for quite a proportion, which is similar to Wilkins et al. [31] study about shaping factors of Southern Ocean microbial assemblage, and this might suggest that Crenarchaeota is related to Antarctic environment. In addition, Actinobacteria and Gammaproteobacteria were two highly abundant classes accounting for $29.4 \%$ and $16.4 \%$ of total dataset, respectively, which is in accordance with the result of Mosier Annika et al. study that Actinobacteria (42\%) dominated in the surface ice community and Gammaproteobacteria (52\%) dominated in the deep ice community [32].

The Venn diagram showed that the four species of Antarctic fish shared many OTUs and also each of them had many unique OTUs which indicates that some similar microbiota lives in intestine of the four species because of the same living conditions, while different organisms are parasitic in the gut for the reason of different life habits and species. The PCA and heatmap presented no obvious difference or similarity in the intestinal microbial community composition among the four species, and we are speculating that bacterial communities in Antarctic fish intestinal tract might have an influence on the physiology of digestion as at present there is no evidence for it.

\section{Conclusion}

The diet and the environment affect the intestinal microbiota of fish and mammals $[33,34]$. In the present study, individuals of each species harbored not really similar intestinal bacterial communities, and gut bacterial communities among the four species were also not absolutely different, suggesting that the common bacterial communities in Antarctic fish 


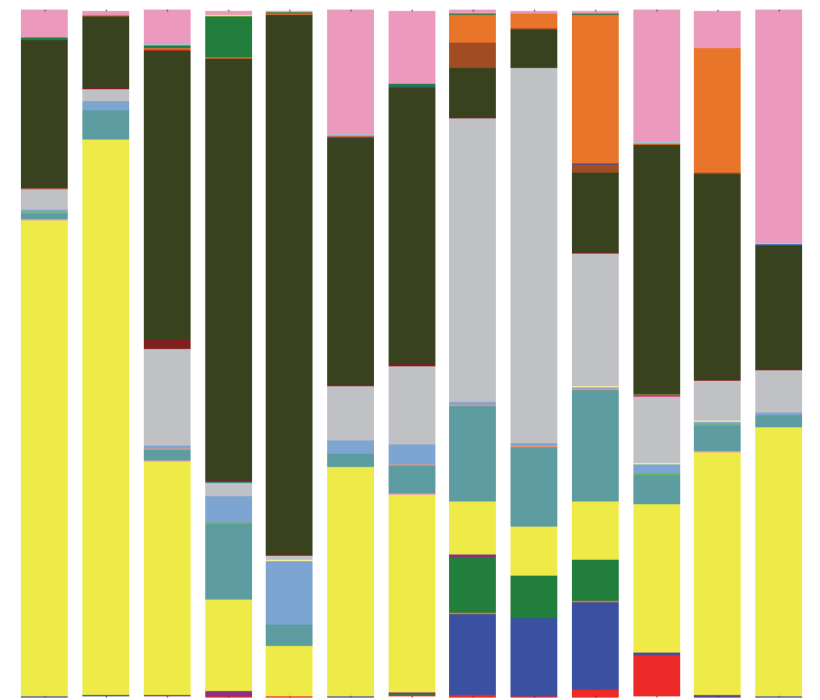

Tb1 Tb2 Tb3 Tb4 Pb1 Pb2 Ch1 Ch2 Ch3 Ch4 Ch5 Ga1 Ga2
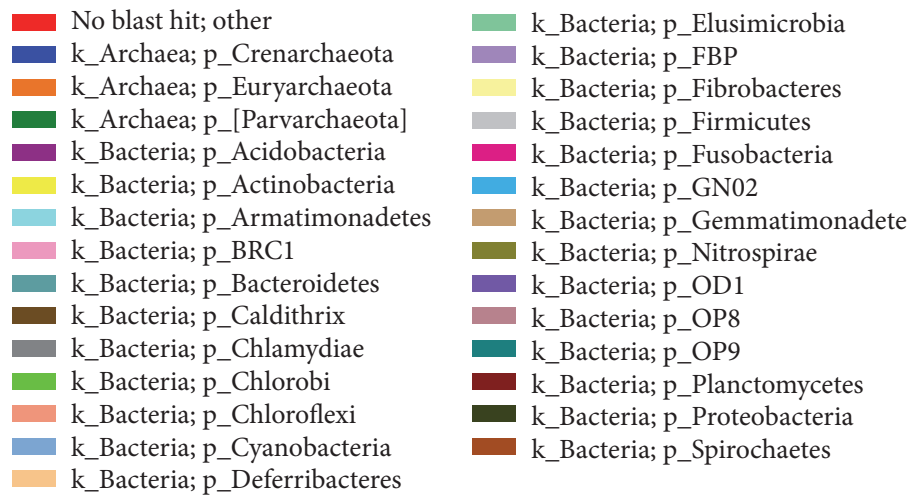

FIGURE 2: The bacterial community composition in different samples.

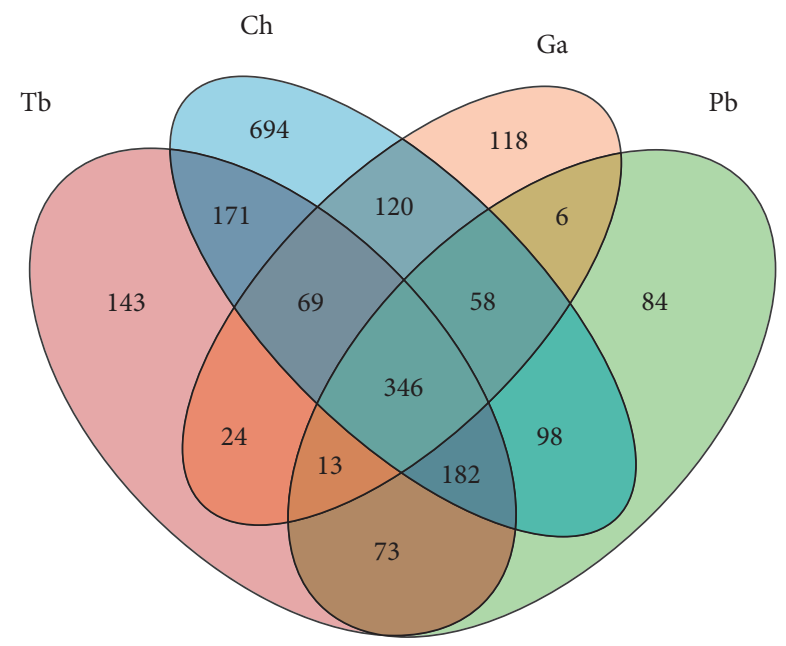

FIgURE 3: The numbers of common and unique OTUs presented in the four species of Antarctic fish.

intestinal tract might contribute to the fish survival ability in extreme Antarctic environment, while the different bacterial communities might be related to the living habits such as diet, depth, and location. All of these results may contribute to

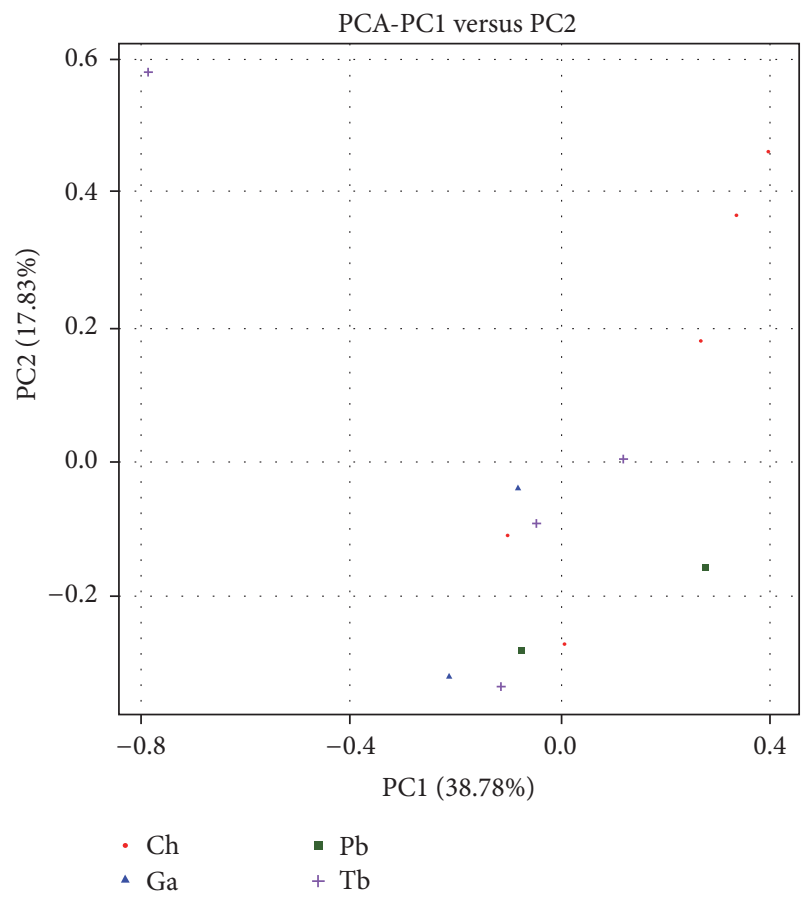

FIgURE 4: Principal Components Analysis (PCA) of the dissimilarity between the microbial samples. 


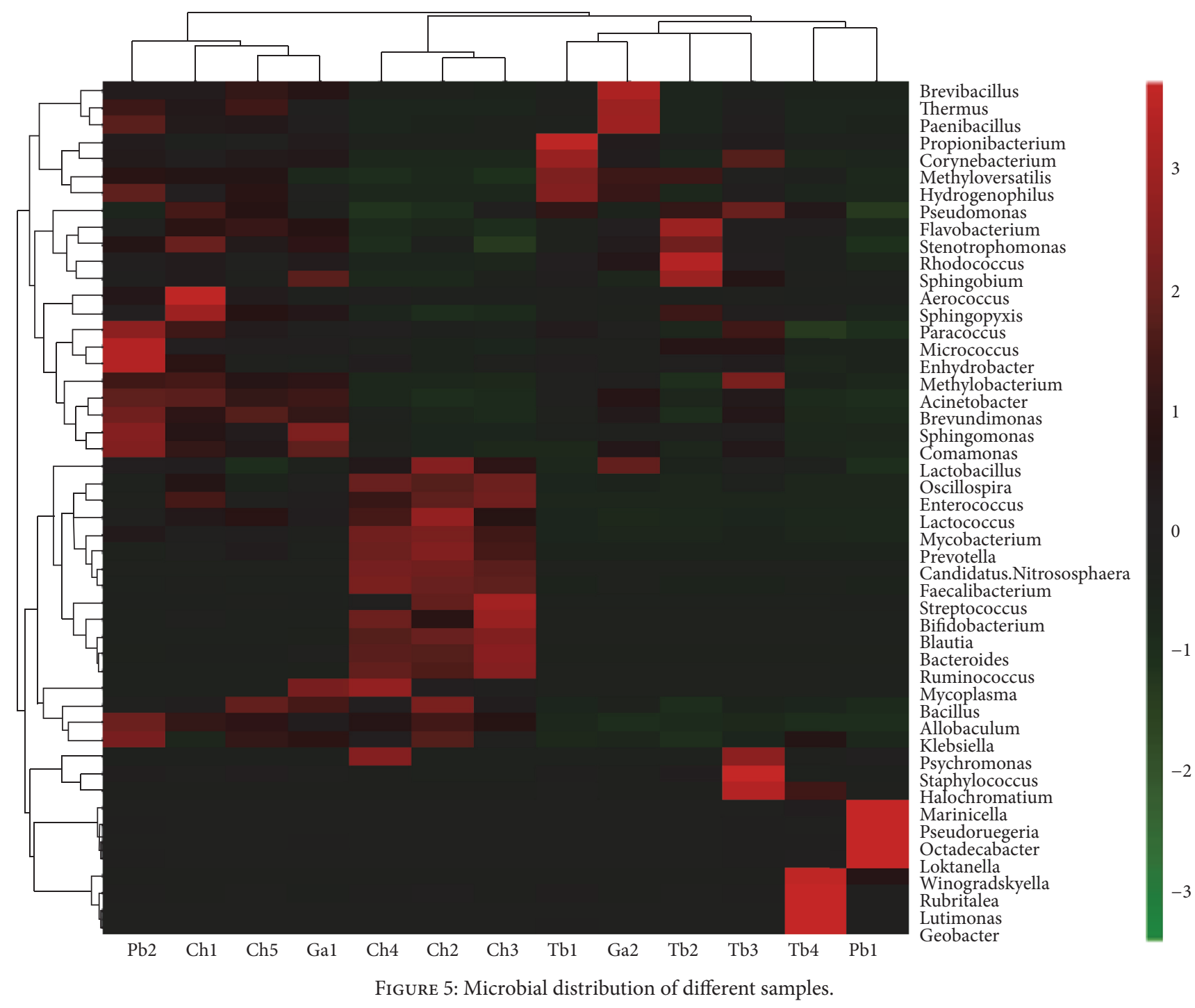

the further study of the relationship between the intestinal bacterial communities and physiological characteristics of Antarctic fish.

\section{Disclosure}

The authors alone are in charge of the content and writing of the paper.

\section{Competing Interests}

The authors declare that there is no conflict of interests regarding the publication of this paper.

\section{Acknowledgments}

This work was supported by the Special Fund for AgroScientific Research in the Public Interest (201203018) and the Chinese National Antarctic Research Expedition
(CHINARE2014-01-06). The authors report no conflict of interests.

\section{References}

[1] J. T. Eastman, "The nature of the diversity of Antarctic fishes," Polar Biology, vol. 28, no. 2, pp. 93-107, 2005.

[2] E. Robinson and W. Davison, "The Antarctic notothenioid fish Pagothenia borchgrevinki is thermally flexible: acclimation changes oxygen consumption," Polar Biology, vol. 31, no. 3, pp. 317-326, 2008.

[3] B. A. Buckley and G. N. Somero, "CDNA microarray analysis reveals the capacity of the cold-adapted Antarctic fish Trematomus bernacchii to alter gene expression in response to heat stress," Polar Biology, vol. 32, no. 3, pp. 403-415, 2009.

[4] C. Verde, E. Parisi, and G. di Prisco, "The evolution of thermal adaptation in polar fish," Gene, vol. 385, pp. 137-145, 2006.

[5] G. di Prisco, J. T. Eastman, D. Giordano, E. Parisi, and C. Verde, "Biogeography and adaptation of Notothenioid fish: hemoglobin function and globin-gene evolution," Gene, vol. 398, no. 1-2, pp. 143-155, 2007. 
[6] M. Ansaldo, C. M. Luquet, P. A. Evelson, J. M. Polo, and S. Llesuy, "Antioxidant levels from different Antarctic fish caught around South Georgia Island and Shag Rocks," Polar Biology, vol. 23, no. 3, pp. 160-165, 2000.

[7] G. E. Hofmann, B. A. Buckley, S. Airaksinen, J. E. Keen, and G. N. Somero, "Heat-shock protein expression is absent in the antarctic fish Trematomus bernacchii (family nototheniidae)," Journal of Experimental Biology, vol. 203, no. 15, pp. 2331-2339, 2000.

[8] L. Bargelloni, S. Marcato, L. Zane, and T. Patarnello, "Mitochondrial phylogeny of notothenioids: a molecular approach to Antarctic fish evolution and biogeography," Systematic Biology, vol. 49, no. 1, pp. 114-129, 2000.

[9] M. Benedetti, G. Martuccio, D. Fattorini et al., "Oxidative and modulatory effects of trace metals on metabolism of polycyclic aromatic hydrocarbons in the Antarctic fish Trematomus bernacchii," Aquatic Toxicology, vol. 85, no. 3, pp. 167-175, 2007.

[10] C. E. Franklin, W. Davison, and F. Seebacher, "Antarctic fish can compensate for rising temperatures: thermal acclimation of cardiac performance in Pagothenia borchgrevinki," Journal of Experimental Biology, vol. 210, no. 17, pp. 3068-3074, 2007.

[11] D. Amelio, F. Garofalo, D. Pellegrino, F. Giordano, B. Tota, and M. C. Cerra, "Cardiac expression and distribution of nitric oxide synthases in the ventricle of the cold-adapted Antarctic teleosts, the hemoglobinless Chionodraco hamatus and the redblooded Trematomus bernacchii," Nitric Oxide, vol. 15, no. 3, pp. 190-198, 2006.

[12] E. Pisano, F. Mazzei, N. Derome, C. Ozouf-Costaz, J.-C. Hureau, and G. Di Prisco, "Cytogenetics of the bathydraconid fish Gymnodraco acuticeps (Perciformes, Notothenioidei) from Terra Nova Bay, Ross Sea," Polar Biology, vol. 24, no. 11, pp. 846852, 2001.

[13] N. Borghesi, S. Corsolini, and S. Focardi, "Levels of polybrominated diphenyl ethers (PBDEs) and organochlorine pollutants in two species of Antarctic fish (Chionodraco hamatus and Trematomus bernacchii)," Chemosphere, vol. 73, no. 2, pp. 155160, 2008.

[14] M. Ghanbari, W. Kneifel, and K. J. Domig, "A new view of the fish gut microbiome: advances from next-generation sequencing," Aquaculture, vol. 448, pp. 464-475, 2015.

[15] A. M. Larsen, H. H. Mohammed, and C. R. Arias, "Characterization of the gut microbiota of three commercially valuable warmwater fish species," Journal of Applied Microbiology, vol. 116, no. 6, pp. 1396-1404, 2014.

[16] E. Ringø, S. Sperstad, O. F. Kraugerud, and Å. Krogdahl, "Use of $16 \mathrm{~S}$ rRNA gene sequencing analysis to characterize culturable intestinal bacteria in Atlantic salmon (Salmo salar) fed diets with cellulose or non-starch polysaccharides from soy," Aquaculture Research, vol. 39, no. 10, pp. 1087-1100, 2008.

[17] N. H. Salzman, H. de Jong, Y. Paterson, H. J. M. Harmsen, G. W. Welling, and N. A. Bos, "Analysis of $16 S$ libraries of mouse gastrointestinal microflora reveals a large new group of mouse intestinal bacteria," Microbiology, vol. 148, no. 11, pp. 3651-3660, 2002.

[18] T. Magoč and S. L. Salzberg, "FLASH: fast length adjustment of short reads to improve genome assemblies," Bioinformatics, vol. 27, no. 21, pp. 2957-2963, 2011.

[19] J. G. Caporaso, J. Kuczynski, J. Stombaugh et al., "QIIME allows analysis of high-throughput community sequencing data," Nature Methods, vol. 7, no. 5, pp. 335-336, 2010.
[20] R. C. Edgar, B. J. Haas, J. C. Clemente, C. Quince, and R. Knight, "UCHIME improves sensitivity and speed of chimera detection," Bioinformatics, vol. 27, no. 16, pp. 2194-2200, 2011.

[21] P. D. Schloss, S. L. Westcott, T. Ryabin et al., "Introducing mothur: open-source, platform-independent, communitysupported software for describing and comparing microbial communities," Applied and Environmental Microbiology, vol. 75, no. 23, pp. 7537-7541, 2009.

[22] V. Carginale, F. Trinchella, C. Capasso, R. Scudiero, and E. Parisi, "Gene amplification and cold adaptation of pepsin in Antarctic fish. A possible strategy for food digestion at low temperature," Gene, vol. 336, no. 2, pp. 195-205, 2004.

[23] N. L. Ward, B. Steven, K. Penn, B. A. Methé, and W. H. Detrich III, "Characterization of the intestinal microbiota of two Antarctic notothenioid fish species," Extremophiles, vol. 13, no. 4, pp. 679-685, 2009.

[24] P. A. Cziko, C. W. Evans, C.-H. C. Cheng, and A. L. DeVries, "Freezing resistance of antifreeze-deficient larval Antarctic fish," Journal of Experimental Biology, vol. 209, no. 3, pp. 407420, 2006.

[25] T. Li, M. Long, F.-J. Gatesoupe, Q. Zhang, A. Li, and X. Gong, "Comparative analysis of the intestinal bacterial communities in different species of carp by pyrosequencing," Microbial Ecology, vol. 69, no. 1, pp. 25-36, 2015.

[26] A. Vergara, M. Franzese, A. Merlino et al., "Structural characterization of ferric hemoglobins from three Antarctic fish species of the suborder notothenioidei," Biophysical Journal, vol. 93, no. 8, pp. 2822-2829, 2007.

[27] H. A. Campbell, K. P. P. Fraser, C. M. Bishop, L. S. Peck, and S. Egginton, "Hibernation in an Antarctic fish: on ice for winter," PLoS ONE, vol. 3, no. 3, Article ID e1743, 2008.

[28] P. Franchini, C. Fruciano, T. Frickey, J. C. Jones, and A. Meyer, "The gut microbial community of Midas cichlid fish in repeatedly evolved limnetic-benthic species pairs," PLOS ONE, vol. 9, no. 4, Article ID e95027, 2014.

[29] L. Ye, J. Amberg, D. Chapman, M. Gaikowski, and W.-T. Liu, "Fish gut microbiota analysis differentiates physiology and behavior of invasive Asian carp and indigenous American fish," ISME Journal, vol. 8, no. 3, pp. 541-551, 2014.

[30] A. Spor, O. Koren, and R. Ley, "Unravelling the effects of the environment and host genotype on the gut microbiome," Nature Reviews Microbiology, vol. 9, no. 4, pp. 279-290, 2011.

[31] D. Wilkins, E. Van Sebille, S. R. Rintoul, F. M. Lauro, and R. Cavicchioli, "Advection shapes Southern Ocean microbial assemblages independent of distance and environment effects," Nature Communications, vol. 4, article 2457, 2013.

[32] C. Mosier Annika, A. E. Murray, and C. H. Fritsen, "Microbiota within the perennial ice cover of Lake Vida, Antarctica," FEMS Microbiology Ecology, vol. 59, no. 2, pp. 274-288, 2007.

[33] K. E. Sullam, S. D. Essinger, C. A. Lozupone et al., "Environmental and ecological factors that shape the gut bacterial communities of fish: a meta-analysis," Molecular Ecology, vol. 21, no. 13, pp. 3363-3378, 2012.

[34] C. Schwab, B. Cristescu, J. M. Northrup, G. B. Stenhouse, and M. Gänzle, "Diet and environment shape fecal bacterial microbiota composition and enteric pathogen load of grizzly bears," PLoS ONE, vol. 6, no. 12, article e27905, 2011. 

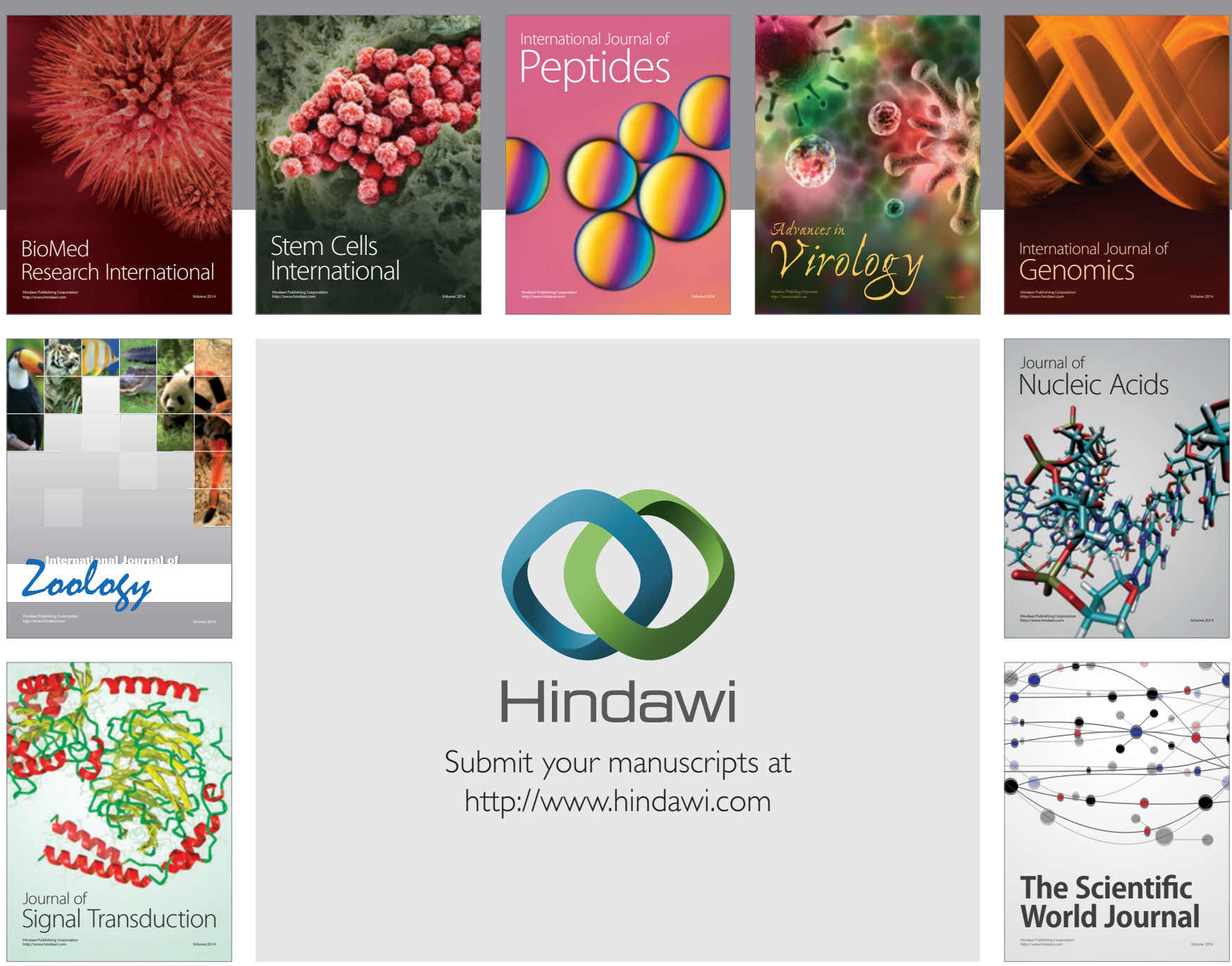

Submit your manuscripts at

http://www.hindawi.com
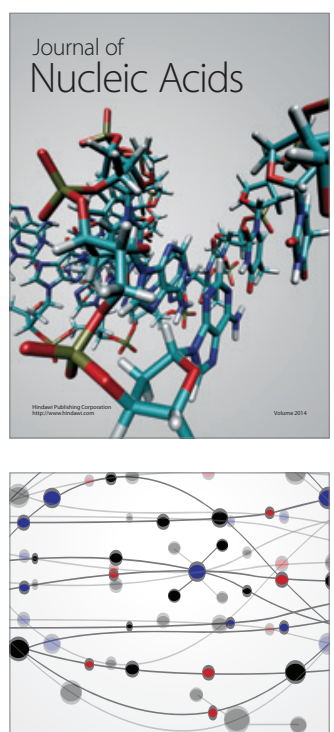

The Scientific World Journal
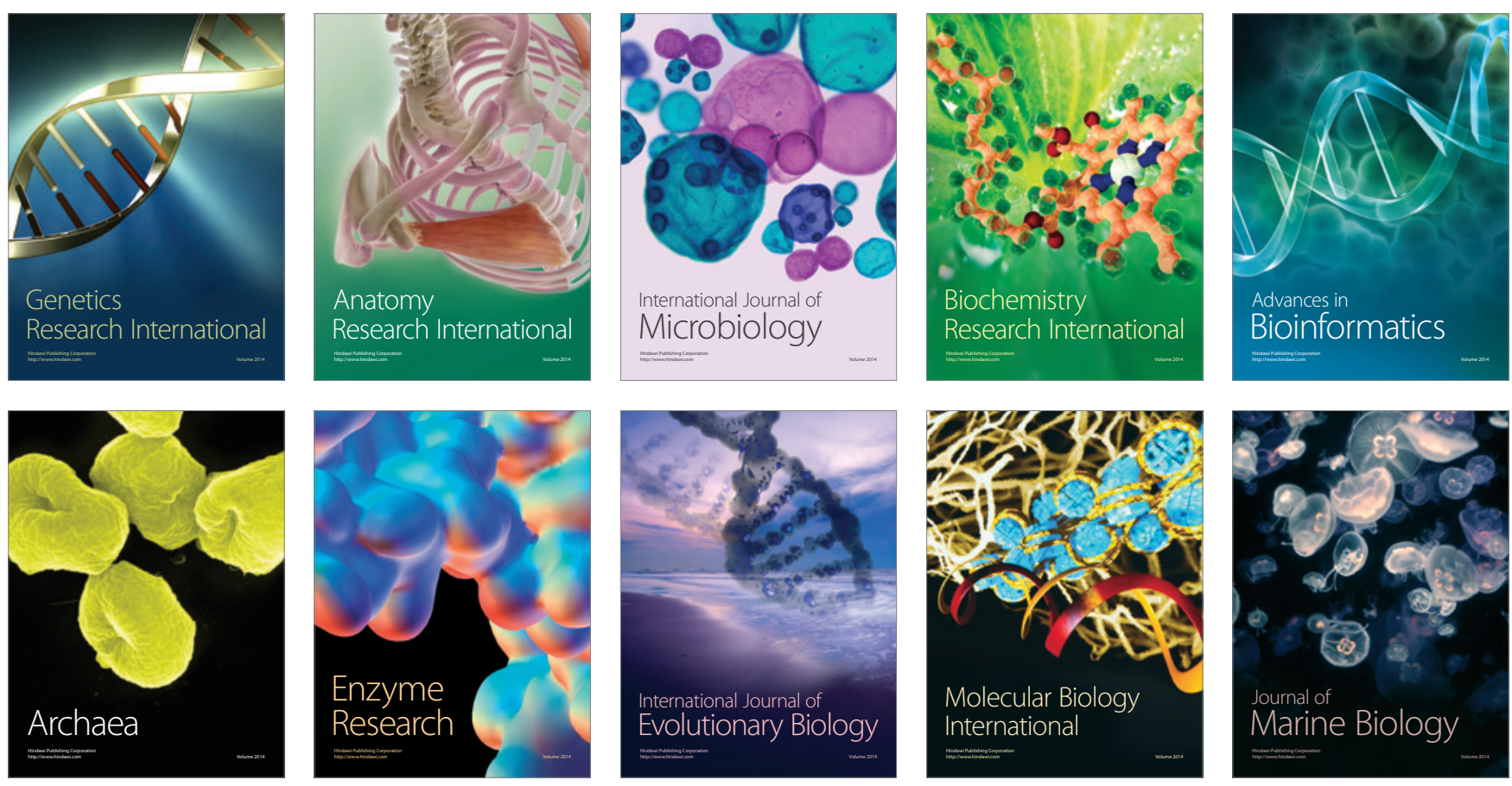